\title{
Essais
}

ESSAIS

Revue interdisciplinaire d'Humanités

17 | 2021

Quels lieux pour les morts?

\section{Comparaison et réflexions sur l'image de Yanluo}

Comparison and reflections on the image of Yanluo Places

Jing Han

\section{OpenEdition}

1 Journals

Édition électronique

URL : https://journals.openedition.org/essais/8474

DOI : 10.4000 /essais. 8474

ISSN : 2276-0970

Éditeur

École doctorale Montaigne Humanités

Édition imprimée

Date de publication : 15 avril 2021

Pagination : 39-48

ISBN : 979-10-97024-09-3

ISSN : 2417-4211

Référence électronique

Jing Han, «Comparaison et réflexions sur l'image de Yanluo », Essais [En ligne], 17 | 2021, mis en ligne le 27 avril 2021, consulté le 18 janvier 2023. URL : http://journals.openedition.org/essais/8474 ; DOI : https://doi.org/10.4000/essais.8474 


\section{Comparaison et réflexions sur l'image de Yanluo}

\section{HaN Jing}

En Chine, si quelqu'un va mourir, on peut dire " il va voir le roi Yanluo ". Pour le peuple chinois, Yanluo, c'est le dieu qui gouverne le monde après la mort. Mais en fait, Yanluo ou bien Yama-raja n'a fait son apparition en Chine qu'avec l'introduction du bouddhisme. Dans le présent travail, nous allons d'abord voir quelques dieux et lieux de la mort avant l'introduction du bouddhisme en Chine, puis nous allons essayer d'analyser l'évolution des images de Yanluo dans les sutras et les fictions après son entrée.

\section{Les dieux et les lieux de mort avant Yanluo}

Avant l'entrée du bouddhisme en Chine, depuis la plus haute antiquité, le culte de la nature et le culte de gui shen 鬼神 (les fantômes et les dieux) ont toujours existé en Chine. Tu bo 土伯 ${ }^{1}$ (littéralement " seigneur du sol»), Bei dou 北斗 ${ }^{2}$ (littéralement " étoiles au nord») et Taishan 泰山 (littéralement « montagne de Tai »), étant divinisés en tant que dieux naturels, étaient associés à la vie et à la mort humaine.

D'après les recherches sur les inscriptions de l'oracle de la dynastie des Shang et les inscriptions sur bronzes de la dynastie de Zhou, dans ces époques (1600-256 avant J.-C.), les gens croyaient que leurs ancêtres entreraient dans la cour céleste et deviendraient les fonctionnaires de l'empereur de jade après la mort, et pourraient donc bénir leurs descendants. Au fil du temps, le concept de vie et de mort s'enrichissait et évoluait constamment. Le changement le plus évident est celui du concept de l'âme. Les gens commençaient à

1 Dans les légendes, Tu bo est gardien de youdu 幽都 (capitale d'obscurité). Avec une tête de tigre, un corps de bœuf, trois yeux et une paire de cornes, il chasse et poursuit les fantômes dans le monde d'après la mort.

2 Bei dou sont les étoiles de la grande casserole. Selon le taoïsme, le dieu de Bei dou est chargé de dissiper le malheur et les malédictions, en même temps que le dieu de Nan dou (étoiles du sud) est chargé de prolonger l'espérance de vie et d'octroyer le bonheur. 
croire que l'âme est composée de deux parties : hun 魂 et $p o$ 魄. Après la mort, « le qi (esprit) de hun va retourner dans le ciel, et la forme (fantôme) de po va descendre sous la terre ${ }^{3}$. Jusqu'aujourd'hui il existe encore une façon d'exprimer l'âme comme "san hun qi po (trois hun et 7 po) ". L'homme vient de la nature et retourne naturellement dans la nature après sa mort. Jusque-là, les Chinois n'imaginaient pas beaucoup la vie après la mort, et le monde obscur restait très ambigu.

Le monde aux yeux du peuple chinois se compose de trois parties : le ciel en haut pour les divinités, la terre au milieu pour les vivants, et l'espace souterrain pour les morts. Puisque l'âme est divisée en deux, le hun est désormais nommé shen 神 (au sens de l'esprit, la divinité), le po est nommé gui 鬼 (fantôme, spectre $)^{4}$. Certains shen 神 peuvent monter dans le ciel, et devenir membres de la cour de l'empereur de jade ; alors où vont tous les gui 鬼? En fait, pour cette question il existe plusieurs notions dans les pensées des Chinois : Huangquan 黄泉 (séjour jaune), Taishan 泰山 (montagne Tai), Fengdu 丰都 (cité de Fengdu), Haoli 蒿里, etc. Plusieurs recherches portent sur ces notions. 5 . Signalons seulement deux points :

- Premièrement, Huangquan est différent de Taishan et de l'Enfer : ces deux dernières sont les résidences dans l'espace ombreux qui imitent le système social du monde vivant avec des institutions similaires, dont la structure est complète. La notion de Huangquan est uniquement dérivée des coutumes funéraires des premiers peuples, et il n'en existe pas un système structuré. Mais il est indéniable que Huangquan est peut-être la première notion concernant l'espace ombreux et le domaine infernal.

- Deuxièmement, les pratiques religieuses autour de la montagne Tai sont anciennes, environ 3000 ans de pratique depuis les Shang jusqu'aux Qing. Avec le temps, s'est établi un culte impérial officiel qui a fait de la montagne Tai l'un des principaux lieux où l'empereur rendait hommage au Ciel (sur le sommet) et à la Terre (au pied de la montagne) lors des sacrifices Fengshan 封禅.

3 《魂气归于天, 形魄归于地。”《礼记》(《li ji 》).

4 《左传”（唐）孔颖达注疏（"zuo zhuan》 [Tang]kongyingda zhu shu). 《以魂本附气, 气 必上浮, 故言魂气归于天。魄本归形, 形既入土, 故言形魄归。圣王缘生事死, 制其祭 祀, 存亡既异, 别为作名, 改生之魂日神, 改生之魄日鬼。”.

5 邵颖涛, 《冥界与唐代叙事文学研究》, 南开大学博士学位论文, 2010 年, 第 9-16 页。(SHAO Yingtao, "L'étude du monde souterrain et la littérature narrative de la dynastie Tang ", Thèse doctorante de l'université de Nankai, 2010, p. 9-16.) ; 范军《佛教地狱观 念与中古叙事 文学》，第 21-25 页 (FAN Jun, «Le concept de l'enfer bouddhiste et la littérature narrative médiévale », p. 21-25) ; 张乡里《黄泉、泰山、蒿里考》, 《贵州民族学院学报 (哲学社会科学版)》2012 年第2期, 第 127-132 页。(ZHANG Xiangli, «Recherches sur Huangquan, Taishan, Haoli ", Journal of Guizhou Nationalities University [édition sur la philosophie et les sciences sociales], 02, 2012, p. 127-132). 
Le statut de la montagne Tai est si important dans les croyances du peuple chinois, non seulement parce que la montagne est naturellement considérée comme le plus proche endroit pour communiquer avec le Ciel, mais aussi parce que le pouvoir culturel de $\mathrm{Qi}$ et $\mathrm{Lu}^{6}$ avait une influence déterminante sur le reste du pays dans leurs époques. L'empire de Qin a unifié la Chine, mais il devait compter sur les intellectuels de Qi et Lu pour la gestion de l'empire $^{7}$. À cette époque, le culte des ancêtres se développait en $\mathrm{Lu}$, le culte de la nature se développait en $\mathrm{Qi}$, et puisque la montagne Tai marquait la frontière entre les États de Qi (au nord du mont) et de Lu (au sud), elle était centre de pratiques religieuses pour les deux. Sans doute avec les efforts des élites de Qi et Lu, la montagne Tai possédait une forte influence culturelle sur tout le pays jusqu'au début de la dynastie des Han.

\section{Les images de Yanluo dans les sutras}

Depuis la dynastie des Han de l'Est, la conviction que les fantômes reviennent aux pieds de la montagne Tai est profondément ancrée dans l'esprit des gens. Pendant ces périodes, avec la traduction des sutras bouddhistes, une divinité exotique, Yanluo (Yama) s'est intégrée peu à peu dans le système du monde souterrain en Chine. La longue et multiple histoire du roi Yama en Chine est un long processus de « sinisation».

Yanluo ou Yanluo Wang, était une translittération de Yama-raja; dans le brahmanisme, Yama était essentiellement un roi du dharma. En tant que premier homme décédé, Yama était en corrélation avec le monde des morts. Alors qu'il est le souverain des enfers, on dit que son royaume se situe également au plus haut des cieux et qu'il se trouve dans la région méridionale du monde. Il a une sœur, nommée Yami, qui a également été identifiée comme la déesse d'une rivière. Yama règne sur les hommes morts, sa sœur sur les femmes mortes. C'est pourquoi parfois on les appelle les " doubles rois yama " (ou " rois jumeaux »).

Le bouddhiste Yama a hérité de son prototype brahmanique ses trois fonctions de gardien du sud, de dieu de la mort et de juge des morts. Et en absorbant la pensée de karma (cause et effet), l'image de Yama évoluait vers le côté effrayant. Il a été vaguement mentionné dans le "Zhe gu shi senlin shu» (Taittirìya Upanisad) que Yama séparait le bon du faux, et dans la «mo nu fa lun " (Manusmrti) il est clairement indiqué que Yama allait torturer les méchants et leur imposer une réincarnation inférieure. Dans le "wangshi shu» (Livre du passé), Yama devient le « juge de l'être humain » et dirige plusieurs

6 Pendant la dynastie de Zhou, le pays se composait de plusieurs états, Qi et Lu en faisaient partie.

7 刘影, 泰山府君与阎罗王更替考, 华东师范大学学报(哲学社会科学版), 1999 (03) : 第 36-41 页 (Liu Ying, "Recherches sur l'alternance entre Taishan Fujun et Yanluo Wang", Journal de l'Université normale de HuaDong (Philosophie et sciences sociales), 03, 1999, p. 36-41). 
couches de l'enfer. Dans les deux épopées de Mahabharata et de Moroyan, Yama avait la peau noire et les yeux rouge, et il était suivi de deux chiens à quatre yeux ${ }^{8}$. A ce stade, Yama n'était plus le gouverneur du bonheur dans le monde du pèlerinage du Rig-Véda et descendait dans l'enfer sombre pour devenir un dirigeant féroce et effrayant. ${ }^{9}$

Faute de trouver un document qui peut nous montrer l'image de Yama en Inde, on peut l'imaginer selon la description des sutras. Voici deux dessins de Yama qu'on trouve au Tibet :

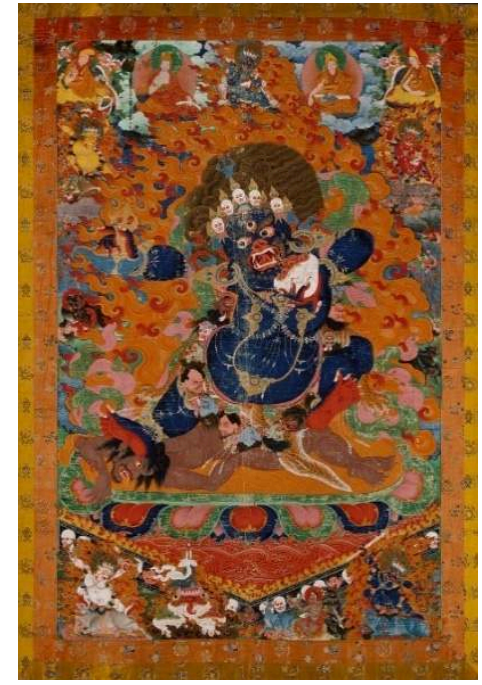

Figure 1 : Yama, fin XVII ${ }^{\text {- début }}$ XVIII ${ }^{\mathrm{e}}$ siècle, Tibet, Metropolitan Museum of Art, New York

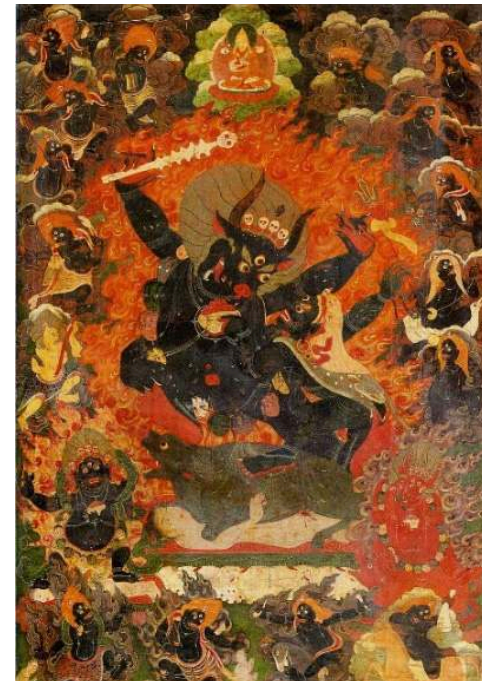

Figure 2 : Yama, XVII siècle, Tibet

\section{Yanluo et son image aux dynasties Wei et Jin, du Nord et du Sud ${ }^{10}$}

Le bouddhisme est introduit en Chine aux alentours des années avant ou après J.-C., en apportant le concept de "ye 业 " (karma, cause et effet) et de "lun hui 轮回》(Samsāra, réincarnation).

8 Marc Tiefenauer, dans son ouvrage "Les enfers indiens : Histoire multiple d'un lieu commun", remarque une coïncidence intéressante : on peut trouver deux chiens dans le mot chinois employé pour traduire le terme bouddhique signifiant l'enfer. Ce caractère "獄 » est constitué de 言 (parole) au centre et flanqué de deux 犬 (chiens).

9 李南.试论摩罗的源与流. 南亚研究, 1991(2) : 71-72 (LI Nan, «Sur la source et le flux de Muoluo ", South Asia Research, 02, 1991, p. 71-72).

10 Les dynasties Wei et Jin (220-420 après J.-C.), les dynasties du Nord et du Sud (420-589 après J.-C.). 
Le mot Diyu 地狱 (enfer, littéralement "prison de terre ») est apparu pour la première fois dans la traduction des sutras par An Shigao dans la dynastie des Han de l'Est : "fo shuo shiba ni li jing" (littéralement sutra des 18 niraya $(\text { enfer })^{11}$ ), "fo shuo zui ye ying bao jiaohua diyu jing" (dicton du Bouddha : les crimes doivent être rapportés aux enfers), "fo shuo gui wen mu lian jing" ("sutra de Mulian enquêté par gui»), etc. Dans le sutra "Fo shuo fenbie shan e suo qi jing" (sutra de la différence entre le bien et le mal), les " cinq voies " ${ }^{12}$ sont mentionnées, dont la cinquième est la voie de Taishan diyu (l'enfer de montagne Tai). Les sutras traduits pendant les dynasties Wei Jin et du Nord et du Sud ont tous adopté la montagne Tai pour indiquer Niraya (l'enfer). La croyance de la montagne Tai était si répandue dans cette période que le bouddhisme devait en profiter pour mieux s'intégrer dans la culture.

Même si le bouddhisme était bien accepté par le peuple chinois à cette époque, la présence de Yanluo dans la littérature n'était pas très fréquente. Seulement dans quelques histoires de Youminglu 幽明录 et de Xuanyanji 轩 辕集, le roi Yama apparaît comme le seigneur de l'enfer. Par exemple, une histoire de Li Tong (conte 263) dans Youminglu se lit comme suit : " Li Tong, originaire de Pucheng, est revenu de la mort en disant : "J'ai vu Fazu, le moine bouddhiste, expliquant Shou leng yan jing 首楞严经 (le Suramgama Sutra) au roi Yama. J'ai également vu le prêtre taoïste Wang fu ${ }^{13}$, entravé, priant Fazu de l'écouter pour ses confessions, pourtant Fazu n'était pas disposé à le faire». " ${ }^{14}$

À cette époque, l'image de Yanluo commençait à entrer dans le monde obscur après mort.

\section{Yanluo et son image depuis les dynasties Sui et Tang}

Selon un sutra traduit dans les périodes des six dynasties ${ }^{15}$, "Chang ahan jing » (Dīrgha Āgama), Yanluo et son entourage ont été punis dans l'enfer pour leur péché, torturés de 3 heures du jour à 3 heures de la nuit. Son statut ne dépassait même pas celui des mortels à cette époque-là.

Mais depuis les dynasties de Sui et Tang, le statut de Yanluo s'améliora beaucoup. "Parmi les histoires concernant l'enfer recueillies dans " Tai ping guang ji », la quantité des histoires concernant Yanluo dépassa celle concernant

11 Différentes écritures bouddhistes contiennent différents dictons par le Bouddha sur les enfers : il y en a quatre, voire six, huit, dix, quinze, dix-huit, trente-six, soixante-quatre, sans limites, et ainsi de suite.

12 Les cinq voies 五道: celles des dieux, des hommes, des condamnés, des e gui 饿鬼 (preta, démon affamé), et des animaux. Voir Marc Tiefenauer, "Les enfers indiens : Histoire multiple d'un lieu commun ", p. 112-113.

13 Wang Fu était un célèbre taoïste dans la dynastie de Jin.

14 Le texte original : 《蒲城李通, 死云: 见沙门法祖为阎罗王讲首楞严经; 又见道士王浮身 被锁械, 求祖忓悔, 祖不肯赴。”

15 Les six dynasties désignent les dynasties des Wei Jin et les dynasties du Nord et du Sud. 
"le Seigneur de montagne Tai $»^{16}$, et on peut croire qu'à cette époque dans les croyances populaires, Yanluo aurait pu avoir une plus grande influence que le seigneur de la montagne Tai.

Dans le parcours de sinisation de Yama en Chine, une ouvre aurait pu beaucoup contribuer : "Fo shuo shiwang jing " (sutra des dix rois/sutra de la révélation faite au roi Yama) est "l'un des premiers ouvrages qui mentionne les dix rois de l'enfer ${ }^{17}$. Stephen Teiser a estimé que "le système des dix rois de l'enfer était une combinaison de deux concepts : d'une part, le concept indien de karma et samsara et, d'autre part, le concept chinois de relation clanique et la bureaucratie impériale ${ }^{18}$.

D'après le sutra des dix rois, en descendant dans l'enfer, de la première à la $7^{\mathrm{e}}$ semaine, à chaque période de 7 jours les morts doivent passer devant un roi de l'enfer pour être jugé et condamné : le $1^{\text {er }}$ septième jour devant Qinguang wang, le $2^{\mathrm{e}}$ devant Chujiang wang, le $3^{\mathrm{e}}$ devant Songdi wang, le $4^{\mathrm{e}}$ devant Wuguan wang, le $5^{\mathrm{e}}$ devant Yanluo wang, le $6^{\mathrm{e}}$ devant Biancheng wang, le $7^{\mathrm{e}}$ devant Taishan wang, puis le centième jour on doit passer devant Pingzheng wang, et le jour d'un an devant Dushi wang, enfin au bout de trois ans devant Wudaolunzhuan wang (le roi chargé de la réincarnation des 5 voies).

Le culte des dix rois correspondait aux 10 étapes de deuil traditionnelles chinoises de "sept sept ", " centième jour ", " un an » et " trois ans ». Cela est lié aux coutumes funéraires avec dix cérémonies de sacrifices. Les fantômes des morts devaient être jugés selon un horaire fixe, ses proches vivants devaient donc faire des cérémonies pour prier les dix rois de réduire leurs souffrances dans l'enfer, et pour qu'ils puissent monter dans le ciel quand les dix cérémonies auraient été achevées.

La croyance en dix rois est restée stable jusqu'au présent, et sur les dessins représentant les dix rois il y avait une claire tendance à la sinisation de l'apparence : visage, habit, chapeaux, etc., comme ce que nous montre cette image ${ }^{19}$ :

16 Voir 范军, 唐代小说中的阎罗王——印度地狱神的中国化. 华侨大学学报(哲学社会 科学版, 2007(01) : 第 92-98 页 (FAN Jun, "Le roi yanluo dans les fictions de la dynastie des Tang - la sinisation du dieu de l'enfer indien ". Journal of Chinese Overseas University [Philosophy and Social Sciences Edition], 01, 2007, p. 92-98).

17 Stephen Teiser, Scripture of the Ten Kings, University of Hawaii Press, 1994, p. 8. Les premiers copies du texte existantes sont rédigées en 908 après J.-C. Pourtant, la première référence aux dix rois, faite par Daoxuan, date de 664.

18 Ibid., p. 3.

19 Source gallica.bnf.fr / Département des Manuscrits. 


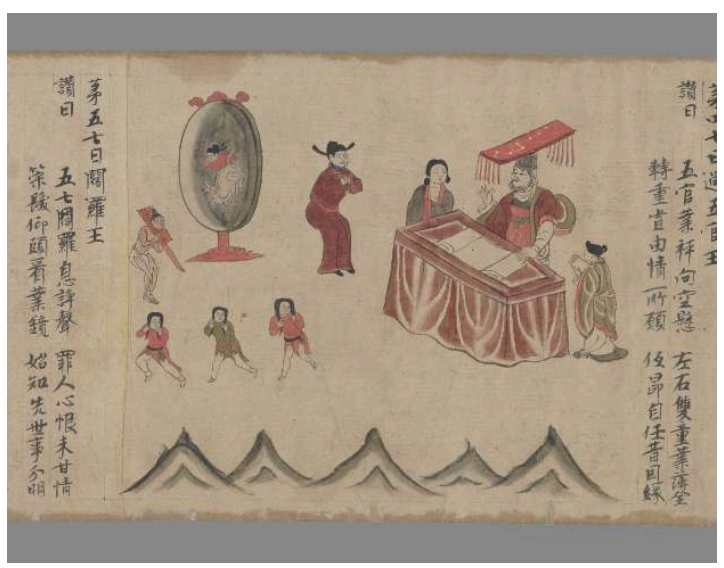

Figure 3 : le $5^{\mathrm{e}}$ sept jours avec Yanluo wang

On peut voir que la nature du roi Yama a changé. Dans son origine indienne, Yama était plutôt un homme divinisé, on donnait des traits nonhumains à un être pour le rendre divin. En Chine, c'est l'inverse, on imagine les dieux sous la forme de l'être humain, on donne des traits humains aux immortels. Par conséquent, l'apparence du roi Yanluo est tout à fait celle d'un homme de l'époque, à l'instar des ministres du monde vivant. Non seulement l'apparence de Yanluo a changé, ses caractères et ses fonctions changeaient également avec le temps; on pourrait le constater surtout dans les fictions et les légendes.

\section{Les images de Yanluo dans les fictions et les légendes}

Les peuples des six dynasties étaient pieux. Mais depuis l'époque des dynasties Sui et Tang, la croyance concernant l'enfer semble s'être clairement sécularisée : dans les créations littéraires du thème, il y avait moins de sens religieux. Les créateurs avaient diverses intentions : exprimer l'admiration, faire réfléchir à la vie réelle ou faire la satire de la corruption. Ainsi le contenu des fictions devenait-il très enrichissant.

Dans les idées du peuple, puisque Yanluo est devenu un seigneur en Chine, sa position devait également être détenue par un $\mathrm{Chinois}^{20}$. Yanluo fut donc plus un fonctionnaire qu'une personne spécifique pour les chinois. C'est pourquoi dans la littérature et dans les légendes folkloriques, nombreux sont les personnages qui étaient le roi Yanluo. Depuis les dynasties Sui et Tang jusqu'aux dynasties Ming et Qing, il y avait beaucoup de célébrités qui ont assumé la fonction de Yanluo:

20 范军, 唐代小说中的阎罗王一印度地狱神的中国化. 华侨大学学报(哲学社会科 学版, 2007 (01) : 第 92-98 页 (FAN Jun, "Le roi yanluo dans les fictions de la dynastie des Tang - la sinisation du dieu de l'enfer indien ". Journal of Chinese Overseas University [Philosophy and Social Sciences Edition], 01, 2007, p. 92-98). 
HaN Qinhu 韩摛虎 ${ }^{21}$, Kou Zhun 寇准 ${ }^{22}$, HaN Qi 韩琦 ${ }^{23}$, FAN Zhongyan 范 仲淹 ${ }^{24}$, BAO Zheng 包拯 ${ }^{25}$, sont les plus connus. Il y avait également les Yanluo wang dont on ne connait pas les identités.

Dans les fictions des dynasties des Sui et des Tang, il est enregistré dans “Sui Shu. Han Qinhu, Zhuan 隋书.韩擒虎传》(livre de Sui, biographie de Han Qinhu) qu'avant sa mort :

La mère de son voisin trouvait que la garde d'honneur sous la porte de Han Qinhu était somptueuse, comparable à celle du roi, elle était curieuse et interrogea, l'un parmi eux répliqua : " je viens pour accueillir le roi. "; très vite, la garde d'honneur disparut. L'autre jour, un homme sévèrement malade, paniqué, vint devant la porte de Qinhu, et demanda de voir le roi, les gardiens demandèrent : " quel roi ? ", " le roi Yanluo " dit-t-il, les gardiens allaient le châtier, HAN Qinhu les arrêta et dit : " je suis parvenu à devenir le pilier du pays en vivant, et si je devenais le roi Yanluo, je n'aurais plus de regret. " Et après, il fut malade au lit pour quelques jours et mourut. ${ }^{26}$

Les héros de ces fictions, s'ils étaient amis ou proches d'un fonctionnaire de l'enfer, bénéficieraient de la grâce ou d'avantages dans le monde souterrain. ${ }^{27}$ S'il s'agissait d'un empereur, même le roi Yanluo devait se plier devant lui. ${ }^{28}$

Ces histoires reflètent les caractéristiques des croyances du peuple des dynasties Sui et Tang : une construction de la divinité par l'humanité.

\section{Dans les fictions depuis la dynastie des Song}

Kou Zhun, étant droit et honnête, était choisi par le peuple pour devenir le roi Yanluo. Dans une fiction, sa concubine préférée Xitao lui dit avant la mort :

Je parlais très peu, c'est parce que je craignais de révéler le secret de l'espace ombreux. Puisque je vais mourir aujourd'hui, cela n'est rien de le révéler : Vous allez être le roi Yanfuti (Yanluo) ${ }^{29}$.

21 HaN Qinhu (538-592 après J.-C.), grand général de la dynastie Sui.

22 Kou Zhun (961-1023 après J.-C.), ministre et poète célèbre de la dynastie Song.

23 HAN Qi (1008-1075 après J.-C.), ministre et militaire célèbre de la dynastie Song.

24 FAN Zhongyan (989-1052 après J.-C.), ministre et poète célèbre de la dynastie Song.

25 BAO Zheng (999-1062 après J.-C.), ministre célèbre de la dynastie Song.

26 Le texte original : “其邻母见擒虎门下仪卫甚盛有同王者, 母异而问之, 其中人曰: ‘我 来迎王。'忽然不见, 又有人疾笃, 忽惊走至擒虎家曰: ‘我欲谒王。’左右问曰: ‘何王也?’ 答曰: ‘阎罗王。”擒虎弟子欲挞之, 擒虎止之曰: ‘生为上柱国, 死作阎罗王。死已足 矣。’因寝疾数日竞卒, 是年五十五。”.

27 Voir plus dans «广异记.邓成c (Deng Cheng, Guangyiji)», «宣室志. 崔君 (Cui jun, Xuanshizhi)».

28 Voir «唐太宗入冥记》(Tang tai zong ru ming ji, littéralement TaiZong dans l'enfer), fragment d'un manuscrit DunHuang S2630, on décrit l'expérience de l'empereur TangTaiZong. Accusé par ses frères morts, il devait descendre dans l'enfer pour être jugé par le roi Yanluo en tant qu'âme vivante.

29 Le texte original : «吾向不言, 恐泄阴理:今欲去, 言亦无害。公当为世主者阎浮提王也». 
Le roi Yanluo le plus connu à cette époque et même dans les époques qui la suivent, c'est BAO Zheng, connu sous le nom de Bao Gong 包公 (Seigneur Bao); il est célèbre pour son intégrité et son respect des lois, d'où son autre surnom de Bao Qingtian 包青天 (le juge Bao). Dans «Song Shi. Bao Zheng, Zhuan 宋史.包拯传”(Histoire de Song. Biographie de BAO zheng), même les femmes et les enfants connaissaient son nom ; on l'appelait BAO shizhi (une fonction comme officier). Le peuple de la capitale disait : « il existe deux endroits où on ne peut pas jouir des avantages avec les relations, chez le roi Yanluo et chez le seigneur Bao $»^{30}$.

Étant honnête et strict dans l'application des lois, Bao Zheng est naturellement le meilleur choix pour être le roi Yanluo aux yeux du peuple. Le peuple chinois apprécie beaucoup Xianshibao 现世报 (c'est-à-dire, recevoir les châtiments de ses mauvaises actions dans cette vie même), et avec Bao Zheng en tant que Yanluo on pourrait gagner la justice auprès de lui si on avait subi de l'injustice pendant la vie.

Dans la dynastie des Qing, Pu Songling (1640-1715) avec sa fameuse œuvre "Liao Zhai zhiyi 聊斋志异 " (histoires étranges d'un pavillon solitaire) a largement influencé la vision du peuple chinois concernant l'espace ombreux. Yanluo a pris des images très différentes dans ses histoires. Dans certaines histoires, les vivants pouvaient également accéder temporairement aux fonctions de Yanluo, et l'origine de ces représentants de Yanluo n'était plus les grands nobles ou seigneurs, mais plutôt le peuple de la couche inférieure ou des hommes faisant des études. Par exemple dans une histoire, "Yanluo ", le représentant qui remplissait la fonction de Yanluo pour enquêter sur CAO Cao 曹操 ${ }^{31}$, était le bachelier Li Zhongzhi, dont le caractère était honnête et franc. Le choix des héros par Pu Songling peut refléter un idéal esthétique de l'auteur : les gens de la couche inférieure peuvent avoir de meilleures qualités que les nobles.

L'image de la plupart des Yanluo était positive, ils respectaient strictement les lois de l'espace ombreux et jugeaient des affaires avec justice, protégeaient les innocents contre les officiels corrompus et les hommes méprisables. Pourtant, dans certaines histoires, Yanluo pouvait également être cruel, avide et trompeur, comme dans l'histoire de «XI Fangping 席方平".

\section{Quelques réflexions sur l'image de Yanluo}

Au cours de l'introduction d'une religion étrangère dans un pays, entre cette religion et les religions indigènes, il y a certainement des conflits, ce qui les obligent à s'influencer, se combattre et se fondre. L'image de Yanluo a été

30 Le texte original : 《关节不到, 有阎罗包老》。

31 CaO Cao (538-592 après J.-C.), politicien, militaire et homme de lettres, il est le fondateur du royaume de Wei, l'un des trois royaumes de cette époque. 
influencée au fil du temps par plusieurs courants de pensées : le bouddhisme, le taoïsme, le confucianisme, et les croyances folkloriques, ces courants s'influençant entre eux mais sans former un ensemble homogène.

Malgré une évolution depuis plus d'un millénaire, les images de Yanluo sont toujours variées. Mais la tendance est claire : ce qui était divinisé en Inde est dédivinisé en Chine ; Yanluo n'est plus divin, il a pris des traits et des caractères humains.

En un certain sens, c'est là l'esprit humaniste du peuple chinois : on croit plutôt à l'homme qu'aux dieux. Et sur l'image de Yanluo, nous pouvons voir à quoi le peuple chinois s'intéresse : la justice.

Enfin, bien que Yanluo soit très présent dans l'imaginaire grâce à l'iconographie et aux contes populaires, le roi Yanluo n'a pas de temple qui lui soit dédié, seulement quelques rares sanctuaires où il est honoré en même temps que neuf autres rois de l'enfer, ou avec le bodhisattva Dizangwang. C'est peutêtre parce que dans la nature, l'homme cherche la lumière et à échapper à l'obscurité. Yanluo, étant seigneur de l'espace ombreux, fait naturellement peur aux gens au lieu de les faire rêver.

HaN Jing

Doctorante à Université de Wuhan cristal_cool@126.com

\begin{abstract}
Résumé
En tant que composante importante du système de croyances chinois, des légendes du monde souterrain se sont développées sur une très longue période. Les dieux concernant la mort se sont succédés l'un après l'autre et quelquefois se sont mélangés et ont fusionné entre eux. Ainsi les images concernant ce monde et les dieux de ce monde restent-elles toujours très variées. Dans la première partie du travail, nous parlons de quelques dieux et conceptions de la mort avant l'introduction du bouddhisme en Chine, et puis nous essayons d'analyser les images de Yanluo (le roi Yama) dans les sutras et les fictions après son introduction en Chine. Enfin nous voyons que l'évolution de ses images est un processus de dédivinisation.
\end{abstract}

\title{
Mots clés
}

Roi Yama, Yanluo, Bouddhisme, sutra, divinisé, humanisé.

\begin{abstract}
As an important part of Chinese belief system, the legends of the underground world have developed for a very long time. The gods related to death appears one after another, and sometimes interact and fuse together. As a result, the images of this world underground and the gods in it are very rich and diverse. In the first part of our work, we will talk about some gods and concepts of death before the introduction of Buddhism into China, and then we will try to analyze the images of King Yang Luo in sutras and fictions after Buddhism entered into China, and we will see that the change of the images is a process of humanization.
\end{abstract}

\section{Keywords}

King Yama, Yanluo, Buddhism, sutra, sanctify, humanise. 